\title{
Sudden Death of an Infant due to Endocardial Fibroelastosis - Case Report
}

\author{
R. H. A. I. Rathnaweera ${ }^{1}$
}

\begin{abstract}
Endocardial fibroelastosis (EFE) is an idiopathic disorder of the tropical and subtropical regions of the world that is characterized by the development of restrictive cardiomyopathy. It is characterized by proliferation of both elastic and collageneous fibers within the endocardium, causing diffuse or localized thickening. A three month old baby was admitted to a local hospital in Galle, with a history of sudden onset shortness of breathing for one day. Baby was found to be in a state of heart failure and died within few hours after admission. At the post-mortem examination, heart was found to be enlarged with dilated left ventricle. The endocardium of both ventricles was markedly thickened. Histopathology showed a thick layer of collagenous fibrous tissue and elastin fibres confirmed by Vangisen Elastin stain. The cause of death was given as endocardial fibroelastosis. The underlying mechanisms of myocardial fibrosis remain unclear. It is hypothesized that infectious, inflammatory and nutritional processes are involved in this process. This case highlighted the importance of special histology staining methods when arriving at the cause of death.
\end{abstract}

Key words: infant, sudden death, endomyocardial fibroelastosis, restrictive cardiomyopathy

\section{INTRODUCTION}

Endocardial fibroelastosis (EFE) is an idiopathic disorder of the tropical and subtropical regions of the world that is characterized by the development of restrictive cardiomyopathy (1). It is characterized by proliferation of both elastic and collageneous fibers within the endocardium, causing diffuse or localized thickening. This leads to endocardial stiffness and severely reduced contractility of the involved ventricles.

This is a condition that continues to arouse interest because of its unfavorable prognosis. The cases of endocardial fibroelastosis reported in the literature are generally of small children (2).

Its presentation in newborns is considered rare. Number of cases reported in Sri Lanka is very few.

\section{CASE REPORT}

A three month old baby was admitted to a local hospital in Galle, with a history of sudden onset shortness of breathing for one day. He was apparently well prior to this incident. No history of fever, cough or refusal of feeding. No significant ill health in the past as well. No consanguinity among the parents of this family. This baby was the first child of this family and it was a normal vaginal delivery when the mother was 26 years old. Antenatal history was uncomplicated. Baby was found to be in a state of heart failure on admission and despite ward management, his condition deteriorated rapidly. Cause for the heart failure was not diagnosed and few hours later, baby died. An inquest was held and a post-mortem was ordered.On general examination, no congenital abnormalities 
RATHNAWEERA R. H. A. I., Sudden death of an infant due to endocardial fibroelastosis - Case report. Sri Lanka Anatomy Journal (SLAJ), 1(2): 43-46, 2017

were seen and the face was found to be congested. No injuries were seen. Body weight was $5.3 \mathrm{~kg}$. Brain appears unremarkable. Both lungs were congested and squeezing reveals a fine froth. Kidneys and liver also found to be congested. Heart was enlarged with dilated left ventricle. The endocardium of both ventricles was markedly thickened with the involvement of the papillary muscles and the chordae tendinae (Figure 1,2,3). The myocardium was normal. The microscopic sections done from several areas of both the right and the left ventricle showed a thick layer of collagenous fibrous tissue and elastin fibers confirmed by Verhhoeff Elastin stain (Figure 4, 5, 6). The cause of death was given as endocardial fibroelastosis.

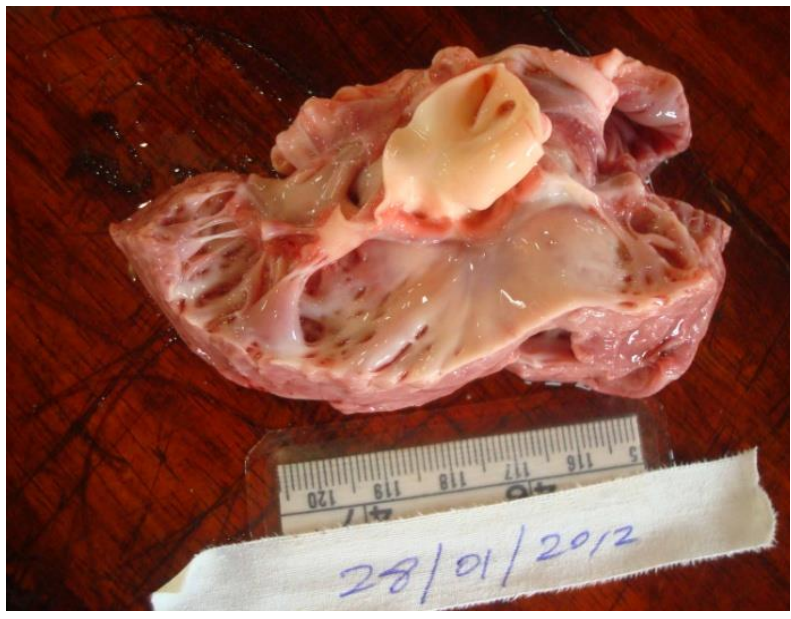

Figure 1: Thickened endocardium of the right ventricle

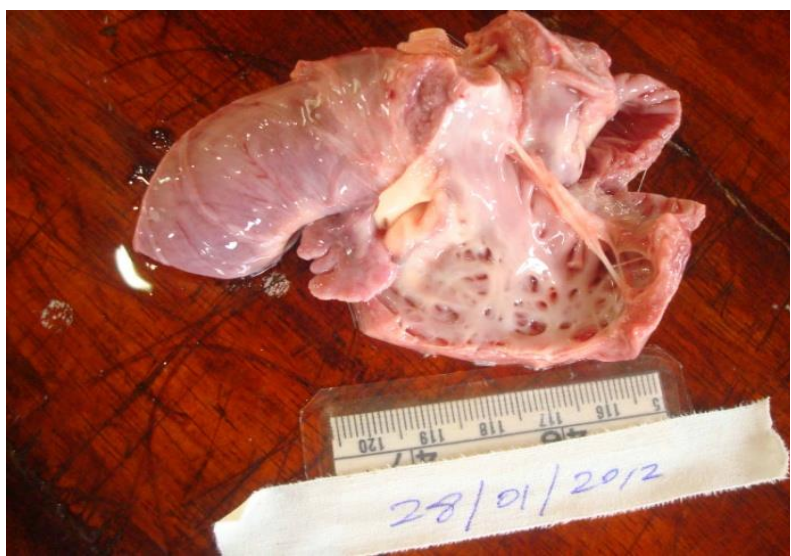

Figure 2: Thickened endocardium of the left ventricle

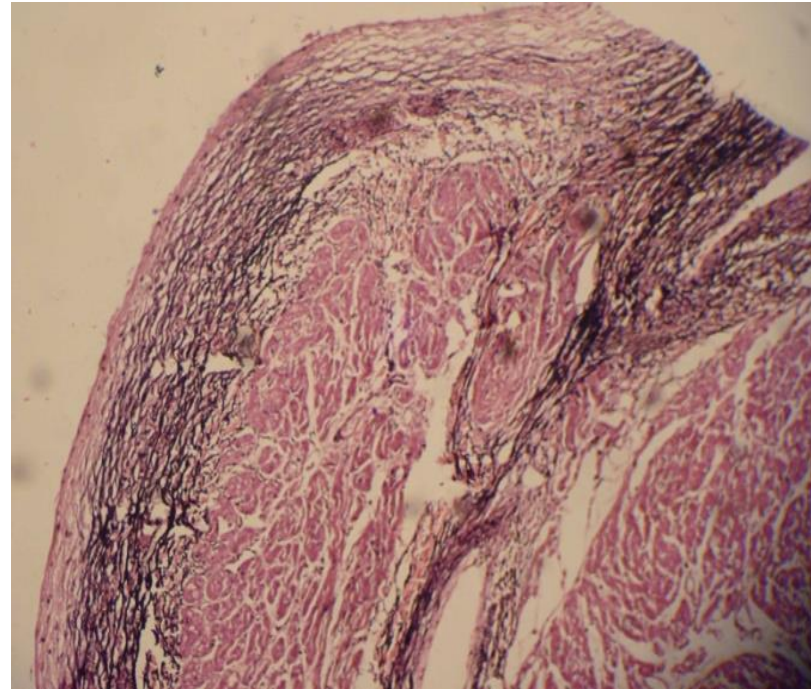

Figure 3: Verhhoeff Elastin stain of the endocardium- lower (x 10) magnification

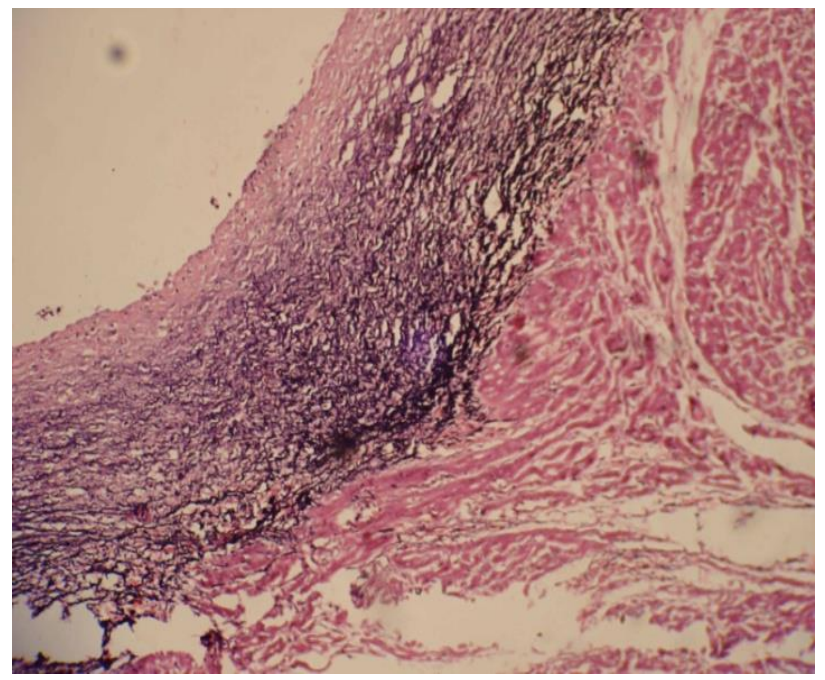

Figure 4: Verhhoeff Elastin stain of the endocardium-higher (x40) magnification

\section{DISCUSSION}

Endocardial fibroelastosis is a rare disorder in newborns, accounting for no more than $1-4 \%$ of total congenital heart disease. It occurs one in every 5000-6000 live births (1). Around $80 \%$ of patients present with congestive heart failure within the first year of life. The distinction into primary and secondary endocardial fibroelastosis has recently been challenged and according to more recent thinking, EFE is simply a reaction to chronic myocardial stress (3). This stress may be 
due to congenital disorders of the vessels and or valves, to viral agents, or to genetic causes determining dysfunction of the myocardium (4). In general, fibrosis in cardiac tissue has been mainly linked to increased level of a cytokine, transforming growth factor- $\beta 1$. The underlying mechanisms of myocardial fibrosis in this specific entity remain unclear. Hypotheses include infectious, inflammatory and nutritional processes as causes (3). EFE is frequently associated with concomitant parasitic infections (eg, helminthes) and their attendant eosinophilia, although the role of parasitic infections and/or the eosinophil remains speculative. The development of EFE as a sequel to toxoplasma-related myocarditis has also been described, as has a relationship of malarial infection to development of EFE. However, no specific organism has been consistently associated with EFE (5).

The endocardial fibrosis develops in the foetus too, the endocardium is the layer most sensitive to injury, being the last to be supplied by the coronary arteries. Many studies have shown that endocardial smooth muscle cells exposed to chronic stress, proliferate and undergo a transition to fibroblasts, producing both collagen and

elastin (6). It is not yet clear how this occurs, but it would seem to be similar to occurrences in other organs. Patchy fibrosis of the endocardial surface of the heart leads to reduced compliance and, ultimately, restrictive physiology as the endomyocardial surface becomes more generally involved. Endocardial fibrosis principally involves the apices of the right and left ventricles and may affect the atrioventricular valves mainly by tethering the papillary muscles, leading to tricuspid and mitral regurgitation. Ventricular stiffness along with atrioventricular valvular regurgitation results in atrial enlargement, which has been linked to atrial arrhythmias such as atrial fibrillation. Fibrosis also reduces conduction velocity, impairs activation pattern and may provide the substrate for wave breaks and reentry (7).

The earliest changes of EFE are not well described because most patients do not present with symptoms until relatively late in the clinical course. Olsen described 3 phases of EFE (8). The first phase involves eosinophilic infiltration of the myocardium with necrosis of the sub endocardium and a pathologic picture consistent with acute myocarditis. This is reportedly present in the first 5 weeks of the illness. The second stage, typically observed after 10 months, is associated with thrombus formation over the initial lesions, with a decrement in the amount of inflammatory activity present. Ultimately, after several years of disease activity, the fibrotic phase is reached, when the endocardium is replaced by collagenous fibrosis. This pathomorphologic schema is not observed uniformly and has not been consistently supported by other investigators.

EFE is most frequently observed in the socially disadvantaged and in children and young women. These groups frequently have malnutrition and in regions of subSaharan Africa where the disease is most prevalent, the typical diet is high in a tuber called cassava, which contains relatively high concentrations of the rare earth element Cerium (Ce). The combination of high $\mathrm{Ce}$ levels and hypomagnesemia has been shown to produce EFE-like lesions in laboratory animals (9). This case highlighted the importance of special histological staining methods when arriving at the cause of death. 


\section{REFERENCES}

1. Weinberg T, Himmelfarb AJ. Endocardial fibroelastosis. Bull Johns Hopkins Hosp. 1943; 72: 299.

2. Moller JH, Lucas RV, Adams P, et al. Endocardial fibroelastosis: a clinical and anatomic study of 47 patients with emphasis on its relationship to mitral insufficiency. Circulation. 1964; 30: 759782.

3. Keith JD, Rose V, Manning JA. Endocardial fibroelastosis. In: Keith JD, Rowe RD, Vlad P, eds. Heart Disease in Infancy and Childhood. $3^{\text {rd }}$ ed. New York, NY: MacMillan; 1978: 941-57.

4. Ni J, Bowles NE, Kim YH, et al. Viral infection of the myocardium in endocardial fibroelastosis. Molecular evidence for the role of mumps virus as an etiologic agent. Circulation. 1997; 95:1339.

5. Chen S, Thompson MW, Rose V. Endocardial fibroelastosis: family studies with special reference to counseling. $J$ Pediatr. 1971; 79: 385-92.

6. Maredia N, English K, Greenwood J. Assessment of endocardial fibroelastosis by cardiac MRI. Can J Cardiol. 2008; 24: e33.

7. Stranzinger E, Ensing GJ, Hernandez RJ. MR findings of endocardial fibroelastosis in children. Pediatr Radiol. 2008; 38: 292-296.

8. M J Newbould, G R Armstrong, A J Barson. Endocardial fibroelastosis in infants with hydrops fetalis. J Clin Pathol. 1991; 44: 576-579.

9. Frobel AK, Hulpke-Wette M, Schmidt KG, Laer S. Beta-blockers for congestive heart failure in children. Cochrane Database Syst Rev. 2009; CD007037.

\section{CORRESPONDENCE}

Dr. R. H. A. I. Rathnaweera

Department of Forensic Medicine, University of Ruhuna, Sri Lanka.

E mail-ajithrathnaweera@gmail.com

Received: July 2017

Accepted: August 2017 